\title{
PARA PENSAR EN EL PESO EXTREMADAMENTE BAJO
}

Juan Augusto Perna Contreras, MD*

"Es necesario hacer todo lo posible para garantizar que un niño nazca a término, bien desarrollado y en estado saludable. No obstante, a pesar de todos los cuidados, los niños nacen de manera prematura".

Pierre Budin, The Nursling

Quiero hacer unas reflexiones después de transcurridos diez años desde mi llegada a esta institución a la unidad de recién nacidos. Realizar la transición entre la vida intra y extrauterina es probablemente el evento individual más peligroso que la mayoría encontremos en nuestra existencia. Nuestros cuerpos deben realizar ajustes fisiológicos radicales de inmediato luego del nacimiento, mayores que en cualquier otro período de la vida.

Grandes adelantos en la atención de los recién nacidos de alto riesgo se han llevado a cabo y el resultado final en la mayor parte de los trastornos neonatales complejos es más favorable, fundamentados en la práctica de la neonatología basada en un gran número de estudios clínicos aleatorios y multicéntricos. Aunque mejoraron las tasas de supervivencia, incluso en los individuos más inmaduros, esta población aún representa una gran preocupación por la morbilidad grave y las desventajas del desarrollo neurológico a largo plazo. No todos los niños que están vivos al nacer permanecen así o sobreviven sin sufrir problemas físicos y mentales graves, tal vez incluso devastadores.

Ejemplo de un caso clínico: un perinatólogo atiende a una mujer de 26 años de edad con 24 semanas de gestación de su primer embarazo, concebido mediante fecundación in vitro. Tuvo una expulsión de líquido claro. Se confirma ruptura de las membranas. La actividad uterina se resuelve con administración de líquidos y reposo en cama intrahospitalaria; la vigilancia de la frecuencia cardíaca es tranquilizante. Se administran dos

Médico-Pediatra. Unidad de Recién Nacidos, Hospital de San José. Profesor Asistente, Fundación Universitaria de Ciencias de la Salud. dosis de betametasona y ampicilina profiláctica, dos días después se queja de dolor de espalda baja y se encuentra una dilatación de $4 \mathrm{~cm}$ con un patrón contráctil activo. Su temperatura aumenta a $40^{\circ} \mathrm{C}$ y la frecuencia cardiaca fetal es de 200 por minuto. Se practica cesárea de urgencia. El niño nace limpio y apneico, con peso de $650 \mathrm{gr}$, extremadamente bajo al nacer, con neumonía y sepsis, más asfixia severa.

Seguramente todos creemos que la única opción que tenemos es reanimar a todo recién nacido en la sala de partos, sin importar que tan pequeño sea y continuar el tratamiento médico agresivo en la unidad de cuidado intensivo neonatal. ¿Hasta cuándo los médicos pueden, y los padres eligen, suspender el tratamiento que sostiene su vida?

La decisión de no iniciar la reanimación en la sala de partos es apropiada para condiciones como:

(*Neonatos con gestación confirmada de menos de 23 semanas o peso al nacer menor de $400 \mathrm{~g}$ (los límites de viabilidad han sufrido un cambio considerable en los últimos 30 años y se anticipa que este cambio va a continuar).

*Anencefalia.

*Bebés con trisomias 13 ó 18 confirmadas.

La información actual indica que la reanimación de estos neonatos es muy probable que resulte en fallecimiento o sobrevivencia con severas discapacidades. ${ }^{1}$

Un estudio británico reciente realizado en prematuros extremos mostró que el $49 \%$ de estos recién nacidos que sobreviven sufren secuelas a los 30 meses de edad; el $23 \%$ minusvalías graves que se manifiestan en la obtención de puntuaciones de desarrollo mental inferiores a tres o más desviaciones estándar por debajo de la media, parálisis cerebrales tan graves que el niño no podía caminar y/o ceguera bilateral. ${ }^{2}$ 
Otra investigación sobre la evolución del recién nacido muy prematuro o con un peso al nacer extremadamente bajo, señala que la frecuencia de retrasos en el desarrollo nervioso permanecen constantes desde la década de los 80, lo que indica que, teniendo en cuenta las técnicas y los cuidados actuales, ya se han alcanzado los límites máximos de viabilidad. ${ }^{3}$

Es posible que un niño con prematurez extrema viva después del parto sin datos prenatales de alguna anomalía ni enfermedad específicas, y que su condición médica y su pronóstico sean tan inciertos que los médicos no puedan realizar una valoración de las perspectivas de supervivencia y el pronóstico al momento del nacimiento.

La posición ética en cuanto a lo apropiado de no aplicar medidas de reanimación en la sala de partos o de suspender el tratamiento en algún momento después de la reanimación, ha llevado a los médicos a aplicar procedimientos excesivos a neonatos con muy mal pronóstico, lo que les causa sufrimiento a ellos y a su entorno sin el beneficio correspondiente.

Otros pediatras simplemente se consideran los mejor calificados y únicos responsables para decidir el destino médico de sus pacientes y asumen que los padres están tan influidos por la ansiedad que no podrían tomar buenas decisiones respecto a su hijo. Los padres tienen vínculos naturales únicos de amor y lealtad con un hijo.

¿Existe una obligación legal y ética para reanimar a todos los recién nacidos y continuar el tratamiento hasta la muerte inminente del niño?

¿Son los médicos u hospitales, en lugar de los padres, los que deben tomar las decisiones para omitir la reanimación o el tratamiento de un recién nacido con peso extremadamente bajo?

\section{Referencias}

1. Hack M, Fanarott AA. Outcomes of children of extremely low birthweight and gestational age in the 1990s. Semin Neonatol 2000;106:235-43.

2. International Guidelines for Neonatal Resuscitation: an Except Fron the Guidelines 2000 for Cardiopulmonary Resuscitation and Emergency Cardiovascular Care: International Consensus on Science. Pediatrics 2000; 106 (3):1-16

3. Wood NS, Marlow N, Costeloe K. Neurologic and developmental disability ofter extremely preter birth. EPICCure Study Gropup.N Engl J Med 2000; 433: 78-84.

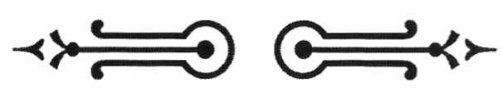

